\title{
Learning Food Quality and Safety from Wireless Stickers
}

\author{
Unsoo Ha, Yunfei Ma, Zexuan Zhong, Tzu-Ming Hsu, Fadel Adib \\ MIT Media Lab
}

\begin{abstract}
Can we sense food quality and safety using wireless signals? In this paper, we explore how we can discover properties of a container's contents without opening it and without any physical contact with its contents. Our idea is to exploit electromagnetic interactions between wireless stickers placed on the container and materials in their immediate vicinity (i.e., inside the container) to determine food quality and safety. We show how a preliminary prototype implementation of our technique enables us to answer questions like: Is a baby formula inside a container pure or tainted? Is the alcohol content of a bottle safe? These capabilities pave way for ubiquitous wireless sensing technologies that can inform their users about the health and safety of their food.
\end{abstract}

\section{INTRODUCTION}

Our health and well-being depend on the safety and quality of the food we eat and drink. Unfortunately, however, consumers today still lack reliable mechanisms to determine the safety and quality of their food. This has led to multiple safety hazards over the past decade. In 2008, Chinese officials discovered that companies had adulterated infant formulas with melamine after the hospitalization of more than 50,000 babies [26]. Similarly, fake alcohol remains an on-going problem in many developing-world countries [13, 17, 24]; in particular, over the past decade, alcohol mixed with methanol has resulted in hundreds of fatalities and numerous cases of blindness in Turkey, Mexico, and China [10, 18].

Indeed, scientists and governments have long recognized the need to assess food quality and safety. The vast majority of existing solutions, however, rely on expensive equipment in specialized food labs [7, 22]. These solutions typically require extracting food samples to perform electro-chemical tests on them for determining bacteria content or impurities. Unfortunately, extracting samples from every purchased item and sending them to food labs for testing is impractical for

Permission to make digital or hard copies of all or part of this work for personal or classroom use is granted without fee provided that copies are not made or distributed for profit or commercial advantage and that copies bear this notice and the full citation on the first page. Copyrights for components of this work owned by others than the author(s) must be honored. Abstracting with credit is permitted. To copy otherwise, or republish, to post on servers or to redistribute to lists, requires prior specific permission and/or a fee. Request permissions from permissions@acm.org.

HotNets-XVII, November 15-16, 2018, Redmond, WA, USA

(C) 2018 Copyright held by the owner/author(s). Publication rights licensed to the Association for Computing Machinery.

ACM ISBN 978-1-4503-6120-0/18/11 . \$15.00

https://doi.org/10.1145/3286062.3286078

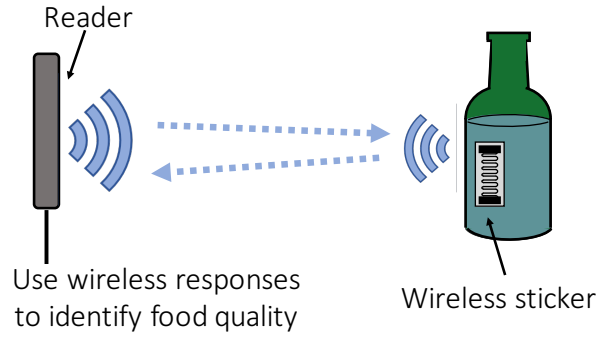

Figure 1-RFIQ's Operation. RFIQ learns food quality and safety by sensing weak coupling between a passive wireless sticker on a container and a container's content. Coupling affects the sticker's response, and can be extracted and analyzed remotely by a reader.

lay consumers. Further, while such techniques are suitable for skip-lot inspections, they are not practical for long-term monitoring applications such as quality detection.

In this paper, we investigate the possibility of determining food safety and quality by relying on cheap, battery-free, wireless stickers. In particular, over the past few years, many industries have moved to replacing bar-codes with batteryfree RFID (Radio Frequency Identification) stickers that are attached to objects. When queried by a wireless device called a reader, these RFIDs power up and respond with a unique identifier, enabling the reader to read and uniquely identify them from distances of few meters. Today's RFIDs are used mainly for product identification and efficient inventory control. Our goal is to leverage these same stickers - which are already placed on billions of products - for learning food quality and safety.

We present RFIQ, a system that can learn food quality and safety by relying on cheap, battery-free RFID stickers without any hardware modifications to the RFIDs. RFIQ operates by extracting and analyzing an RFID's response at the analog level. In particular, an RFIQ reader transmits a wireless signal to power up the RFID on a food container of interest and extracts low-level features from the RFID's response as shown in Fig. 1. It then uses these features to learn different properties about the container's contents, such as baby formula purity and alcohol composition.

RFIQ's basic formulation is inspired by the electromagnetic principles of multi-layered substrates for printed antennas. Specifically, a UHF (Ultra-High Frequency) RFID antenna is typically printed on a flexible plastic substrate [41]. The printed antenna's geometry is optimized to achieve the highest gain (i.e., strongest signal) at the desired frequency of operation (around $950 \mathrm{MHz}$ ) given the substrate material. To improve the gain of these antennas, past research has explored leveraging multi-layer substrates [41], where the antenna's 
interaction with materials is in its immediate vicinity is governed by a concept called "weak coupling".

RFIQ's key realization is that placing an RFID's antenna on a filled container is equivalent to adding another thick substrate layer below its plastic substrate, with which the RFID also couples. Yet, in contrast to past proposals which have designed the antenna geometry and matching circuit in a manner to optimize its response on a multi-layer substrate, such a placement would result in reducing the antenna's efficiency at its desired frequency of operation. This is because the RFID antenna design does not account for the new substrate material. Indeed, this is why it is difficult to power up an RFID when it is placed on a bottle with liquid water $[31,33,46]$. Aside from reducing the gain at the desired frequency of operation, such placement results in tuning its optimal operation bandwidth to a new center frequency that better matches the multi-layer substrate. RFIQ's goal is to discover the new tuning frequency to glean information about a bottle's content.

Realizing this goal still requires addressing multiple challenges. First, the captured RFID signal is not only a function of the coupling effect, but also the location of the RFID in the environment as well as multi-path reflections off different objects in the environment, all of which interfere at the receiver. To overcome this challenge, RFIQ exploits the intrinsic differences between the impact of multipath and that of the "weak coupling" on the measured response. In particular, multipath leads to frequency-selective fading which impacts all the frequencies, while the coupling effect leads to shifting the center frequency of the optimal response. Intuitively, the impact of multipath is similar to fast-varying noise in the frequency domain response of the RFID, while the impact of the weak coupling is more localized at the tuning frequency, as we demonstrate empirically in $\S 3.3$.

Another challenge arises from the ability to discover the tuning frequency and map it to the material contents of a container. On one hand, FCC regulations for consumer electronics limit the bandwidth over which we can power up an RFID to within $26 \mathrm{MHz}$, significantly constraining RFIQ's ability to search for the tuning frequency. On the other hand, even if we could discover the tuning frequency, mapping it to the exact material contents of a container is difficult as it requires solving a complex and under-determined set of Maxwell's equations that govern electromagnetic propagation, as we describe in \$3.2. To overcome these challenges, RFIQ first builds on our recent technique which allows measuring an RFID's response over a wide bandwidth while remaining compliant to FCC regulations [20]. Then, it employs a customized machine learning classifier that can automatically learn the desirable features from the frequency domain response of the RFID and use them for classification.

We built an initial prototype of RFIQ and validated it by conducting 230 experimental trials in two applications. We first test RFIQ's ability to identify baby formula adulteration. We show that RFIQ can identify if a baby formula is tainted with more than $96 \%$ accuracy, while sustaining a false negatives rate is $5 \%$. Second, we consider tainted alcohol identification. We show that RFIQ can identify with $97 \%$ accuracy. Note that in both of these applications, users cannot differentiate between tainted and untainted materials due to lack of visible differences between the container contents.

While these results are promising, we believe they only present a first demonstration of RFIQ's capability as a food quality and safety sensor. In the conclusion, we outline open research questions that need to be answered to transform this primitive into a ubiquitous, cost-effective solution for monitoring food quality and safety.

\section{BACKGROUND AND RELATED WORK}

Researchers have long recognized the need for monitoring food quality and safety. Most existing techniques rely on measuring electrochemical and electrophysical properties [19, $25,37]$. Specifically, scientists extract food samples and place them in direct contact with other chemical reagents and/or specialized sensing circuits (e.g., biotoxin sensors $[8,9,15]$ ), to test how they react to these reagents. This typically requires sending samples to research labs for testing. Hence, it is more suitable for skip-lot inspection where a fraction of the samples is inspected (as is typically done by government agencies) rather than everyday usage by lay consumers.

Given the length of the food lab testing process, recent proposals have considered building small sensing circuits in hope of incorporating them inside food containers [28, $30,35,39]$. These proposals require designing a customized sensor for every different type of food or food property of interest $[35,39]$ or they require coating existing circuits (e.g., LC circuits or RFIDs) with different types of polymers to increase sensitivity to specific materials of interest $[28,30]$. Moreover, many of these sensors still require direct contact with food samples, which can lead to contaminating the food samples and is erosive to their sensing interfaces [29, 44].

Recent advances in wireless transmission and signal processing techniques have led to more general wireless solutions that can classify between different objects. These techniques fall under two main categories: radar-based $[6,42,43]$ and tag-based $[11,38,45]$. The problem with these systems, however, is that they are intrinsically incapable of extracting material properties such as food quality. They either rely on radar reflections to classify between objects based on their shapes [6, 43], or they measure how a wireless signal at a single frequency attenuates while traveling through a material before it reaches a tag in order to deliver simple tasks like distinguishing between whether a cup is empty or filled with water or if the density of the material changes [11,27].

A more powerful class of techniques falls under RF dielectric spectroscopy. RF spectroscopy excites a material of interest by electromagnetic waves over a wide range of frequencies and analyzes how it interacts with these waves to determine material properties like food quality and safety [14, 32]. Unfortunately, however, the technique relies on expensive, bulky equipment such as Vector Network Analyzers (which cost 


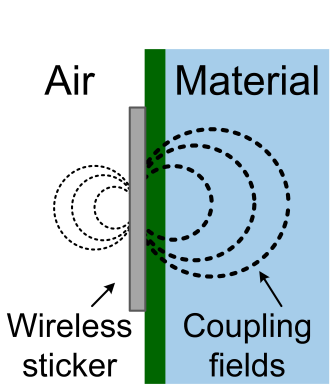

(a) Weak Coupling

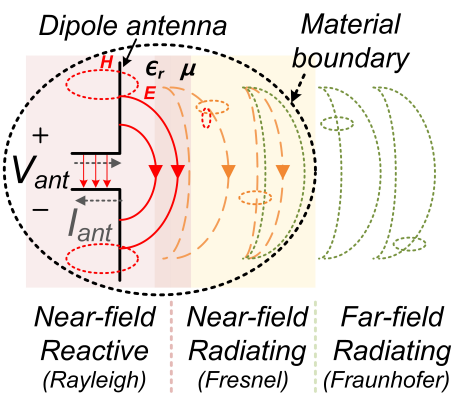

(b) Antenna Radiating Fields

Figure 2-RFIQ's Operation Principle. (a) shows how a wireless sticker couples with material in a container. (b) shows fields of a radiating antenna.

tens to hundreds of thousands of dollars) and requires sophisticated calibration techniques for proper operation [23, 34]. Recent proposals have considered using UWB radios as dielectric spectroscopes [12]; however, they also require extracting liquid samples and placing them in carefully adjusted locations, hence preventing in-situ measurements.

While RFIQ is inspired by these techniques, it introduces new mechanisms that transform cheap, off-the-shelf RFIDs which are already placed on billions of manufactured items - into powerful food sensors without requiring any direct contact with the food material. This enables a cost-effective, scalable approach for in-situ, continuous food monitoring.

\section{RFIQ}

RFIQ is a wireless sensor that can inform lay consumers about the quality and safety of food in its vicinity. To do so, it leverages cheap, passive UHF (Ultra-High Frequency) RFID stickers already attached to the food containers by the manufacturers. It analyzes low-level physical properties of an RFID's response to learn the quality and safety of food in its corresponding container. RFIQ can scan multiple items simultaneously as it incorporates the industry standard (EPC Gen-2) protocol. To map an ID to a particular product, it can leverage an RFID database provided by its manufacturer.

RFIQ operates in two key stages. First, it transmits a wireless signal to power up a passive RFID. When the RFID responds with its identifier, RFIQ decodes the response and extracts from it low-level signal features related to food quality and safety. Second, RFIQ feeds the extracted features into a machine learning classifier to determine the food quality and safety. This involves first selecting the correct classifier based on the food type as determined by the identifier (e.g., alcohol), and then feeding the signal features to that classifier. RFIQ repeats the above process for every RFID in its vicinity, each time selecting the appropriate machine learning classifier to determine the corresponding food quality and safety.

\subsection{Physical Principle: Dielectric Permittivity}

Before delving into the details of RFIQ's operation stages, we describe the basic physical principle that enables it to identify food quality and safety from wireless RFID stickers.

When an RFID is placed in the vicinity of a material, the electromagnetic field of the RFID's antenna is affected by the material contents as shown in Fig. 2(a). This phenomenon is called "weak coupling" [40]. Interestingly, coupling happens even if the RFID's antenna is not in direct contact with the material as shown in the figure. This is because the electromagnetic field of an RFID's antenna occupies a region of space around the antenna.

The magnitude and distribution of this coupling effect are determined by an intrinsic property of the material called dielectric permittivity (denoted by $\varepsilon$ ). A material's permittivity is a function of the electrochemical and electrophysical properties of that material (e.g., molecules and ions in the material). Hence, if the material content changes, so does its dielectric permittivity and subsequently the electromagnetic distribution of the weak coupling.

RFIQ harnesses this property in order to extract features related to food quality and safety. In particular, it tries to sense minute variations in an RFID's response that result from small changes in the permittivity of the material it couples with. Specifically, when an RFID transmits its identifier, the "weak coupling" affects the signal structure of its transmission. ${ }^{1}$ We can represent the response captured by RFIQ as a complex number that has both a magnitude $A$ and a phase $\theta$. The magnitude and phase are functions of both the excitation frequency $f$ and the material's dielectric $\varepsilon$. Mathematically, we can write the response $r$ as $A(\varepsilon(f)) e^{j \theta(\varepsilon(f))}$.

By sensing this response over a wide range of frequencies, RFIQ can learn how small variations in that response are related to various food properties of interest. Finally, it is worth noting that even though RFIQ's technique is inspired by RF dielectric spectroscopy, it does not attempt to directly measure the material's dielectric. ${ }^{2}$ Rather, it relies on the how the phenomenon of weak coupling affects an RFID's response, and learns the impact of dielectric changes on that response to infer the food quality and safety metrics of interest.

\subsection{Analyzing the Received Signal}

Our above discussion focused only on the interaction of the RFID's antenna with its immediate surrounding through weak coupling. In practice, however, when a reader measures an RFID's response, it obtains the signal after it has traveled in space and bounced off different objects. This phenomenon is referred to as multipath, since the receiver may obtain multiple superimposed copies of the RFID's signal after it has reflected off different objects in the environment. Mathematically, the channel due to the wireless propagation in the medium is denoted by $h(t)$, and we can describe the channel of the overall measured response $y(t)$ as:

$$
y(t)=h(t) * r(t)
$$

where $*$ denotes the convolution operator.

So, how can we distinguish between the impact of the material in the direct vicinity of the RFID (e.g., inside the container) and that of the RFID's location with respect to

\footnotetext{
${ }^{1}$ Note that it only affects the shape of the physical signals but not the specific "bits" being transmitted.

${ }^{2}$ Such measurements require a larger bandwidth of operation.
} 
our receiver or other objects in the environment? And, what defines the direct vicinity vs multipath in the environment?

To better understand the difference, consider the illustration in Fig. 2(b). The figure shows an RFID antenna as a dipole with a voltage and a current and distinguishes between three fields: near-field reactive, near-field radiating, and far-field radiating. The near-field reactive decays significantly faster than the far-field. In particular, the near-field is typically limited to within less than a wavelength, while the far-field is dominant at a range larger than two wavelengths.

\subsubsection{The Reactive Near-Field}

The "coupling effect" we described in $\$ 3.1$ happens in the near field. When a voltage is applied across the antenna, it results in a current flowing through the dipole. Ideally, most of the energy delivered to the dipole should be radiated outward toward a receiver in the far field (to maximize the power transmitted). In practice, however, part of the energy is stored or dissipated in the antenna's immediate vicinity.

To understand why this is the case, consider the electric field lines (shown in solid red) between the two arms of the dipole in Fig. 2(b). According to basic antenna theory [16], the two arms of the dipole act like two plates of a capacitor, whose overall capacitance is determined by the electric permittivity $\varepsilon$ of the material between the plates. A larger permittivity leads to larger capacitance, hence a larger amount of energy stored in the near-field.

Similarly, as the current flows through the conductor, it results in a magnetic field (shown in dashed red), whose energy is also stored in the vicinity. The amount of stored magnetic energy is determined by the magnetic permittivity $\mu$ of the material surrounding the conductor. The magnetic and electric energy storage effect can be modeled by a self-inductance $L(\mu)$ and a self-capacitance $C(\varepsilon)$. An antenna resonates when it is excited by a frequency $\omega=1 / \sqrt{L(\mu) C(\varepsilon)}$. This is why when the dielectric in the vicinity of the antenna changes, its optimal frequency of operation changes.

The above discussion is simplified in multiple ways. First, it does not attempt to solve for the exact tuning frequency $\omega . \mathrm{Ob}$ taining an exact analytic solution requires solving Maxwell's equation at medium boundaries, and an analytic solution may be intractable.

Second, from a circuits perspective, the impact of nearfield coupling on the antenna response will also depend on the matching circuit, which is optimized to the antenna's geometry and its typical medium of operation (i.e., air).

Given this complex relationship, the electromagnetic equations do not have a general solution, and a solution is typically obtained through numerical solvers such as the method of moments (MoM). In contrast, RFIQ leverages a learning-based approach as we describe in §3.3. A learning approach is intrinsically more powerful since it can seamlessly account for other factors (e.g., complex antenna geometry, different substrates) without requiring a priori knowledge.

To better understand the impact of the dielectric on the antenna response, we run an experiment where we power

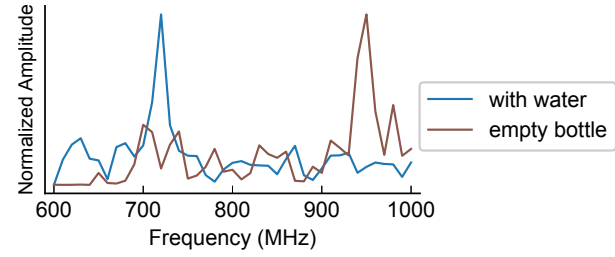

Figure 3-Frequency tuning of an RFID due to material content. We plot the normalized amplitude of the RFID's response versus frequency when the RFID is on an empty bottle (in brown) and a water-filled bottle (dark blue). While an RFID is designed to have optimal response at $950 \mathrm{MHz}$ in air, its optimal frequency shifts to about $720 \mathrm{MHz}$ due to coupling with water.

up and communicate with a commercial passive UHF RFID at different frequencies. The RFID is placed on a container. We measure the amplitude of the RFID response at different frequencies and plot the output in Fig. 3. The figure plots the normalized amplitude as a function of the excitation frequency in the absence (brown) and presence (blue) of water inside the container. The figure shows that while the RFID is designed to have an optimal response around $950 \mathrm{MHz}$ in air, its optimal frequency response shifts to around $720 \mathrm{MHz}$ due to the coupling with water.

\subsubsection{The Radiative Far-Field}

So far, we have discussed the impact of the reactive near-field on the antenna impedance. Recall that the overall channel is also impacted by far field propagation. Specifically, as the RFID's signal travels in space, it reflects off different objects leading constructive and destructive interference, that exhibits in frequency-selective fading. Unfortunately, such fading may null or zero-out the new tuning frequency of the RFID's response, preventing us from detecting it, and potentially leading to classification errors.

To overcome this challenge, RFIQ leverages intrinsic differences in the patterns of frequency-selective fading and nearfield coupling (or antenna tuning). In particular, antenna tuning causes a shift in the center frequency of optimal operation as shown in Fig. 3, while frequency-selective fading causes a high frequency noise. This is because frequency-selective fading occurs at the order of the coherence bandwidth, which varies between 4-20 MHz in indoor environments [36]. For example, in Fig. 3, it would lead to fluctuations in the amplitude of the response, yet we still expect the highest amplitude to remain around the new frequency (tuned to the dielectric). In practice, these intrinsic differences allow RFIQ to achieve high accuracy as we demonstrate qualitatively and quantitatively in the next sections.

\subsection{Feature Extraction \& Classification}

Next, we describe how RFIQ can extract relevant features from the wide bandwidth and use them for classification.

Extracting Features from a Wide Bandwidth. Recall that the dielectric material causes frequency tuning. However, searching for the new tuning frequency is prohibitive for multiple reasons. First, commercial passive RFIDs are optimized to harness power within a specific frequency range (as per the matching circuit) [21]. And second, FCC regulations limit the bandwidth for powering up RFIDs to within $26 \mathrm{MHz}$. 


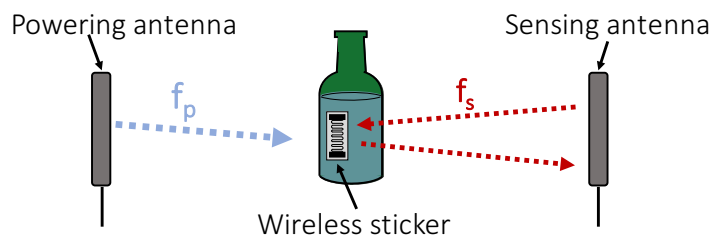

Figure 4-Sensing an RFID's response over a wide bandwidth. RFIQ uses an RFID excitation technique called two-frequency excitation to sense the response over a wide bandwidth. The first frequency is used to deliver power in the ISM band. The second frequency is used to sense changes in the sticker's response (due to the dielectric) over a wide bandwidth.

To overcome this challenge, RFIQ builds on our recent technique, two-frequency excitation [20], which enables powering up an RFID and sensing its response at two different frequencies. At a high level, instead of using the same frequency to deliver power to an RFID and sense its response, the technique employs two frequencies as shown in Fig. 4. The first frequency is used to power up the RFID within the ISM band, while the second frequency can be used to sense the response within a completely different band, ideally where the dielectric changes are most pronounced. Since the frequencies most affected by dielectric changes are difficult to predict (due to the non-linear nature of "weak coupling"), RFIQ sweeps over a wide range of sensing frequencies and feeds the resulting output into a classifier that can automatically extract the most salient features.

In order to compute the RFID's response at a given sensing frequency, the receiver can correlate the response with a known preamble. In particular, given a known preamble $p_{t}$ of the tag's response in the received signal $y_{t}$, it can obtain an estimate of the overall channel $l_{k}$ at a given sensing frequency $f_{k}$ as $l_{k}=\sum_{t} y_{t} p_{t}^{*}$. Finally, in order to improve the signal-to-interference-and-noise ratio (SINR) of the extracted features, RFIQ performs two additional steps:

- First, to reliably capture the amplitude and phase over hundreds of $\mathrm{MHz}$ of bandwidth, RFIQ averages multiple responses of an RFID over time.

- Second, to deal with the interference from other sources in the environment, RFIQ not only computes the covariance described above, but also the correlation:

$$
\operatorname{corr}_{k}=\sum_{t} y_{t} p_{t}^{*} / \sqrt{\sum_{t}\left|y_{t}\right|^{2} \sum_{t}\left|p_{t}\right|^{2}}
$$

This allows RFIQ to normalize the response by the power of the received signal. The correlation is only close to 1 when there is indeed an RFID response in the measure signal. Hence, RFIQ uses this correlation output as an input to its classifier in order to aid it in learning the robustness of the measured channels at the different frequencies.

Feature Selection and Classification. RFIQ uses the wide bandwidth to train classifiers for learning various food-related metrics. Its classification operates in three steps. The first is deterministic and leverages the RFID's decoded identifier to choose the appropriate classifier (e.g., alcohol vs baby formula classifier). Second, it extracts a feature vector, consisting of normalized amplitude $A^{\prime}(f)$, phase $\theta(f)$ and correlation values $\operatorname{corr}(f)$. Third, it employs a standard gradient boosting tree for each classification task. This machine learning classifier is useful in our context because it is known to perform well for features with sparse information, and it iteratively constructs trees to combine features.

\section{EXPLORATORY STUDY}

\subsection{Prototype Implementation}

Hardware. We implemented our design on USRP N210 software radios [3], and adapted our two-frequency excitation prototype [20]. The radios run the EPC-Gen2 protocol and transmit two frequencies: one high power frequency (at $32 \mathrm{dBm}$ ) inside the UHF ISM band and another low power sensing frequency which is varied within $400-800 \mathrm{MHz}$. At the sensing frequency's receiver, we employ a low-pass filter that eliminates the impact of the power up frequencies and add an LNA to boost the received signal power. The transmission powers are compliant to FCC regulations for consumer electronics [1]. The received signal is sampled (digitized) and sent over Ethernet to a computer for offline processing.

Software. RFIQ's algorithms are implemented in MATLAB and python. The MATLAB component averages every 50 RFID responses to boost the SNR and computes the amplitude and phase at every sensing frequency. It repeats this process over 21 frequencies, spaced at $20 \mathrm{MHz}$ intervals, across RFIQ's $400 \mathrm{MHz}$ frequency span. We use the python implementation of the XGBoost package [4] to implement RFIQ's gradient boosting tree classifiers. The classifier parameters are selected conservatively to prevent over-fitting. We set eta $=0.3$, max_depth $=3$, subsample $=0.5$, and num_boost_round $=128$.

\subsection{Experimental Results}

The goal of our evaluation is to test the feasibility of using RFIQ to learn about food quality. We evaluate RFIQ's operation in a setup similar to Fig. 5. The container of interest is placed in a constrained region (within about $0.5 \mathrm{~m}$ from RFIQ's antennas). The antennas capture the response of the RFID on the container and extract features from it at different sensing frequencies. Across experimental trials, we place the bottle of interest within a bounded region close to RFIQ. This ensures that we can power up the RFID despite the decrease in antenna efficiency. We perform our experiments with commercial off-the-shelf passive UHF RFIDs, the Avery Dennison AD-229r6 tag [2].

Identifying Tainted Alcohol. First, we would like to test RFIQ's ability to identify fake alcohol. Recall that alcohol can be tainted by mixing it with the much cheaper methanol. To simplify the sensing task, we consider only 5 classes of adulteration. The first class is pure alcohol, and the rest have $25 \%, 50 \%, 75 \%$, and $100 \%$ methanol. We prepared mixtures with these compositions. For data collection, we fill a plastic bottle with one of the mixtures and place it in a randomly chosen location in our setup. The bottle has an RFID attached on it. We run RFIQ's sensing and feature extraction algorithms 


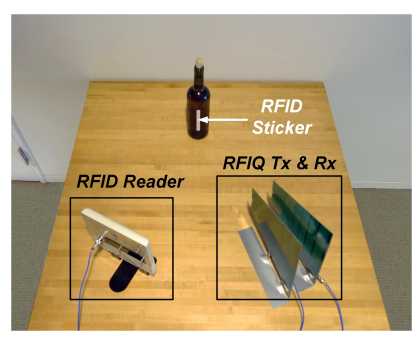

Figure 5-RFIQ's Setup. RFIQ's antennas measure the response of an RFID placed on a bottle. We vary the bottles and their locations across experimental runs.

as described in $\S 3.3$ and $\S 4.1$. For each mixture, we repeat the experiment in 3 different locations and with 10 different bottles (of similar shape $\&$ material). ${ }^{3}$ This provides 150 data points, 30 for each adulteration class. Note that our goal of using different bottles and locations is to test the robustness of the classification to minor environmental changes.

To test RFIQ's ability to distinguish between the different adulteration classes, we performed standard 10-fold crossvalidation. In particular, for each classification task, we divided our dataset into testing set (consisting of 15 trials performed with the same bottle and all 5 classes) and training set (consisting of the remaining 135 datapoints). We repeated the same classification task 10 times, each time with a different bottle, and computed the average accuracy.

Table 1(a) shows our results as a confusion matrix. The different rows represent the actual percentage of methanol tainting, while the different columns represent RFIQ's predicted percentage. The table shows that RFIQ can classify between the different mixtures, with an average accuracy of $97 \%$. Note that the errors were not limited to adjacent classes (as in the $75 \%$ case); we expect this is because while the compositions are varied linearly, the gradient boosting classifier is not guaranteed to be linear. These results indicate the potential of using RFIQ for identifying adulterated alcohol.

Identifying Adulterated Infant Formula. In our second study, we are interested in evaluating RFIQ's ability to identify whether a baby formula has been adulterated. We prepare five different mixtures of baby formula with melamine. Each mixture consists of $100 \mathrm{~g}$ in total, and with varying percentages of adulteration between $0 \%$ and $30 \%$ (i.e., in increments of $10 \mathrm{~g}$ ). We use the Organic Infant formula from Earth's Best [5] as a baseline for pure infant formula.

We perform 80 experimental trials in total, and test our classifier using 5-fold cross-validation, as above. RFIQ's classification accuracy is shown in the confusion matrix of Table 1(b). The different rows of the matrix show the actual percentage of adulteration, while the different columns show RFIQ's prediction of adulteration. The table shows that RFIQ can classify between the different adulteration classes. In particular, across all the adulterated formulas, it only misclassified $1.25 \%$ of them as pure. Its false positive rate (i.e., it classified a pure formula as non-pure) is also $0 \%$. Moreover,

\footnotetext{
${ }^{3}$ We used the same 10 bottles for the different experiments and refilled them every time with a different mixture.
}

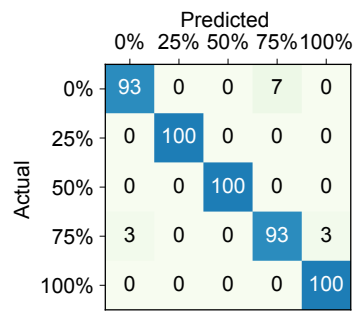

(a) Alcohol Tainting

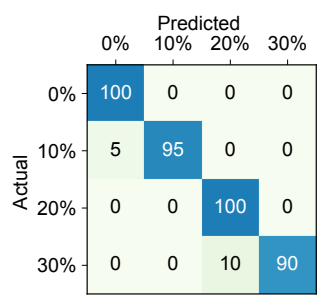

(b) Baby Formula Adulteration
Table 1-RFIQ's Classification Tasks. Different rows of the confusion matrices represent the actual tainting percentage, and different columns represent RFIQ's predicted percentage.

RFIQ is capable of identifying the percentage of contamination with an average accuracy of $96 \%$. This indicates that not only it can be used to identify adulteration, but also has the potential to identify its degree.

\section{OPEN QUESTIONS \& CONCLUSION}

This work presents a new sensing technique can be used to guide consumers against purchasing or consuming hazardous products (such as fake alcohol). While our initial evaluation is promising, multiple questions remain to be answered:

- Environmental Changes: Our initial evaluation shows robustness to limited environmental changes. As the research evolves, we are interested in enhancing robustness to various environmental changes, including container shape, container material, dense multipath, and liquid volume.

- Solution Contents: Our evaluation focused on mixtures of materials of the same state (i.e., solid mixtures or liquid mixtures). An important research question is: Can RFIQ be used to classify and identify solution contents? For example, is it possible to leverage RFIQ to learn sugar content of a liquid or lead content of water?

- Range and Mobility: In our evaluation of RFIQ, we placed the containers of interest in a relatively confined region near RFIQ's sensing antennas. It would be interesting to explore how to miniaturize RFIQ into a handheld device that a user carries in grocery stores or supermarkets.

- Sensitivity and Learning Algorithms: Our implementation focuses on a bandwidth of 400-800 MHz, fixes the types of material and adulterant, and assumes a fixed and discrete number of classes. This simplifies our sensing task into a classification problem and enables sensing food properties whose dielectric changes affect the RFID's response within this bandwidth. We are interested in expanding the bandwidth and exploring other machine learning models to relax these assumptions and improve sensitivity.

As these capabilities expand and grow, we envision that wireless sensing can glean increasingly more sensitive (yet invisible) information about the health and safety of our food and environment, and use this information to guide us on how we may improve our health and well-being.

Acknowledgments. We thank our shepherd Peter Steenkiste, and the anonymous HotNets reviewers for their helpful feedback on the manuscript. This research is partially supported by the MIT Media Lab, NSF, and a Google Faculty Research Award. 


\section{REFERENCES}

[1] Understanding the Fcc Regulations for Low-power, Non-licensed Transmitters. Office of Engineering and Technology Federal Communications Commission, 1993.

[2] Avery Dennison. AD, 2017. http://rfid.averydennison.com/en/home/innovation inlay-designs/AD-229r6-AD-229r6-P.html.

[3] usrp n210. http://www.ettus.com, 2017. ettus inc.

[4] XGBoosting. xgb, 2017. http://xgboost.readthedocs.io.

[5] Organic infant formula. https://www.earthsbest.com/en/products/ category/formula/infant-formula, 2018. Earths Best.

[6] F. Adib, C.-Y. Hsu, H. Mao, D. Katabi, and F. Durand. Capturing the human figure through a wall. ACM Transactions on Graphics (TOG), 34(6):219, 2015

[7] E. C. Alocilja and S. M. Radke. Market analysis of biosensors for food safety. Biosensors and Bioelectronics, 18(5):841-846, 2003.

[8] P. K. Ang, W. Chen, A. T. S. Wee, and K. P. Loh. Solution-gated epitaxial graphene as ph sensor. Journal of the American Chemical Society, 130(44):14392-14393, 2008.

[9] P. Banerjee, S. Kintzios, and B. Prabhakarpandian. Biotoxin detection using cell-based sensors. Toxins, 5(12):2366-2383, 2013.

[10] BBC. Three Russian tourists die in Turkish alcohol poisoning. http://www.bbc.com/news/world-europe-13633313.

[11] R. Bhattacharyya, C. Floerkemeier, and S. Sarma. Low-cost, ubiquitous rfid-tag-antenna-based sensing. Proceedings of the IEEE, 98(9):15931600,2010

[12] A. Dhekne, M. Gowda, Y. Zhao, H. Hassanieh, and R. R. Choudhury. Liquid: A wireless liquid identifier. In ACM MobiSys, 2018.

[13] J. Fullerton. Bathtub booze and knock-off whisky: inside China's fake alcohol industry. The Guardian, 2015. https://www.theguardian.com/sustainablebusiness/2015/sep/16/china-fake-alcohol-industry-counterfeitbathtub-booze-whisky.

[14] K. Grenier, D. Dubuc, P.-E. Poleni, M. Kumemura, H. Toshiyoshi, T. Fujii, and $\mathrm{H}$. Fujita. Integrated broadband microwave and microfluidic sensor dedicated to bioengineering. IEEE TMTT, 57(12):3246-3253, 2009.

[15] W.-D. Huang, H. Cao, S. Deb, M. Chiao, and J.-C. Chiao. A flexible ph sensor based on the iridium oxide sensing film. Sensors and Actuators A: Physical, 169(1):1-11, 2011.

[16] R. C. Johnson and H. Jasik. Antenna engineering handbook. New York, McGraw-Hill Book Company, 1984, 1356 p. No individual items are abstracted in this volume., 1984.

[17] F. Karimi. US warns travelers about tainted alcohol in Mexico. CNN, 2017. http://www.cnn.com/2017/07/27/us/mexico-state-departmentalcohol-warning/index.html.

[18] O. Kurtas, K. Y. Imre, E. Ozer, M. Can, I. Birincioglu, C. Butun, G. S. Kirci, A. Yildirim, S. Kiyak, and R. Yilmaz. The evaluation of deaths due to methyl alcohol intoxication. Biomedical Research, 28(8), 2017.

[19] B. Kuswandi, Y. Wicaksono, A. Abdullah, L. Y. Heng, M. Ahmad, et al. Smart packaging: sensors for monitoring of food quality and safety. Sensing and Instrumentation for Food Quality and Safety, 5(3-4):137146, 2011.

[20] Y. Ma, N. Selby, and F. Adib. Minding the billions: Ultrawideband localization for deployed rfid tags. ACM MobiCom, 2017.

[21] G. Marrocco. The art of uhf rfid antenna design: Impedance-matching and size-reduction techniques. IEEE antennas and propagation magazine, 50(1), 2008

[22] M. McLachlan, R. Hamann, V. Sayers, C. Kelly, and S. Drimie. Fostering innovation for sustainable food security: the southern africa food lab. In The Business of Social and Environmental Innovation, pages 163-181. Springer, 2015.

[23] A. Mellinger. Dielectric resonance spectroscopy: a versatile tool in the quest for better piezoelectric polymers. IEEE TDEI, 10(5):842-861, 2003

[24] J. Miller. 10,000 gallons of bad booze seized from Mexican resorts NY Post, 2017. http://nypost.com/2017/08/14/10000-gallons-of-badbooze-seized-from-mexican-resorts/.
[25] K. Narsaiah, S. N. Jha, R. Bhardwaj, R. Sharma, and R. Kumar. Optical biosensors for food quality and safety assuranceâǍTa review. Journal of food science and technology, 49(4):383-406, 2012.

[26] New York Times. China's Top Food Official Resigns. d- http://www.nytimes.com/2008/09/23/world/asia/23milk.html.

[27] S. D. Nguyen, N. N. Le, P. T. Lam, E. Fribourg-Blanc, C. M. Dang, and $\mathrm{S}$. Tedjini. A wireless sensor for food quality detection by uhf rfid passive tags. In $A T C, 2015$, pages 258-263. IEEE, 2015.

[28] K. G. Ong, J. S. Bitler, C. A. Grimes, L. G. Puckett, and L. G. Bachas. Remote query resonant-circuit sensors for monitoring of bacteria growth: Application to food quality control. Sensors, 2(6):219-232, 2002.

[29] K. G. Ong, J. S. Bitler, C. A. Grimes, L. G. Puckett, and L. G. Bachas. Remote query resonant-circuit sensors for monitoring of bacteria growth: Application to food quality control. Sensors, 2(6):219-232, 2002.

[30] R. A. Potyrailo, N. Nagraj, Z. Tang, F. J. Mondello, C. Surman, and W. Morris. Battery-free radio frequency identification (rfid) sensors for food quality and safety. Journal of agricultural and food chemistry, 60(35):8535-8543, 2012.

[31] X. Qing and Z. N. Chen. Proximity effects of metallic environments on high frequency rfid reader antenna: Study and applications. IEEE TAP, 55(11):3105-3111, 2007.

[32] J. P. Runt, J. J. Fitzgerald, et al. Dielectric spectroscopy of polymeric materials. American Chemical Society, 1997.

[33] H.-W. Son, G.-Y. Choi, and C.-S. Pyo. Design of wideband rfid tag antenna for metallic surfaces. Electronics Letters, 42(5):263-265, 2006.

[34] S. S. Stuchly and M. Matuszewski. A combined total reflectiontransmission method in application to dielectric spectroscopy. IEEE TIM, 27(3):285-288, 1978.

[35] E. L. Tan, W. N. Ng, R. Shao, B. D. Pereles, and K. G. Ong. A wireless, passive sensor for quantifying packaged food quality. Sensors, 7(9):1747-1756, 2007.

[36] M. S. Varela and M. G. Sánchez. Rms delay and coherence bandwidth measurements in indoor radio channels in the uhf band. IEEE TVP, 50(2):515-525, 2001.

[37] A. Vasilescu and J.-L. Marty. Electrochemical aptasensors for the assessment of food quality and safety. $\operatorname{Tr} A C, 79: 60-70,2016$.

[38] J. Wang, J. Xiong, X. Chen, H. Jiang, R. Balan, and D. Fang. Tagscan: Simultaneous target imaging and material identification with commodity rfid devices. ACM MobiCom, 2017.

[39] S.-Y. Wu, C. Yang, W. Hsu, and L. Lin. 3d-printed microelectronics for integrated circuitry and passive wireless sensors. Microsystems \& Nanoengineering, 1:15013, 2015.

[40] F. Yang and Y. Rahmat-Samii. Microstrip antennas integrated with electromagnetic band-gap (ebg) structures: A low mutual coupling design for array applications. IEEE TAP, 51(10):2936-2946, 2003.

[41] H. Yang and N. Alexopoulos. Gain enhancement methods for printed circuit antennas through multiple superstrates. IEEE TAP, 35(7):860863, 1987

[42] Z. Yanzi, Z. Yibo, Y. Z. Ben, and Z. Haitao. Reusing $60 \mathrm{ghz}$ radios for mobile radar imaging. In ACM MobiCom, 2015.

[43] H.-S. Yeo, G. Flamich, P. Schrempf, D. Harris-Birtill, and A. Quigley. Radarcat: Radar categorization for input \& interaction. In Proceedings of the 29th Annual Symposium on User Interface Software and Technology, pages 833-841. ACM, 2016.

[44] J.-Y. Yoon and B. Kim. Lab-on-a-chip pathogen sensors for food safety. Sensors, 12(8):10713-10741, 2012.

[45] J. Zhang and G. Y. Tian. Uhf rfid tag antenna-based sensing for corrosion detection \& characterization using principal component analysis. IEEE TAP, 64(10):4405-4414, 2016.

[46] Y. Zhang, K. Yemelyanov, X. Li, and M. G. Amin. Effect of metallic objects and liquid supplies on rfid links. In IEEE APSURSI'09, 2009. 